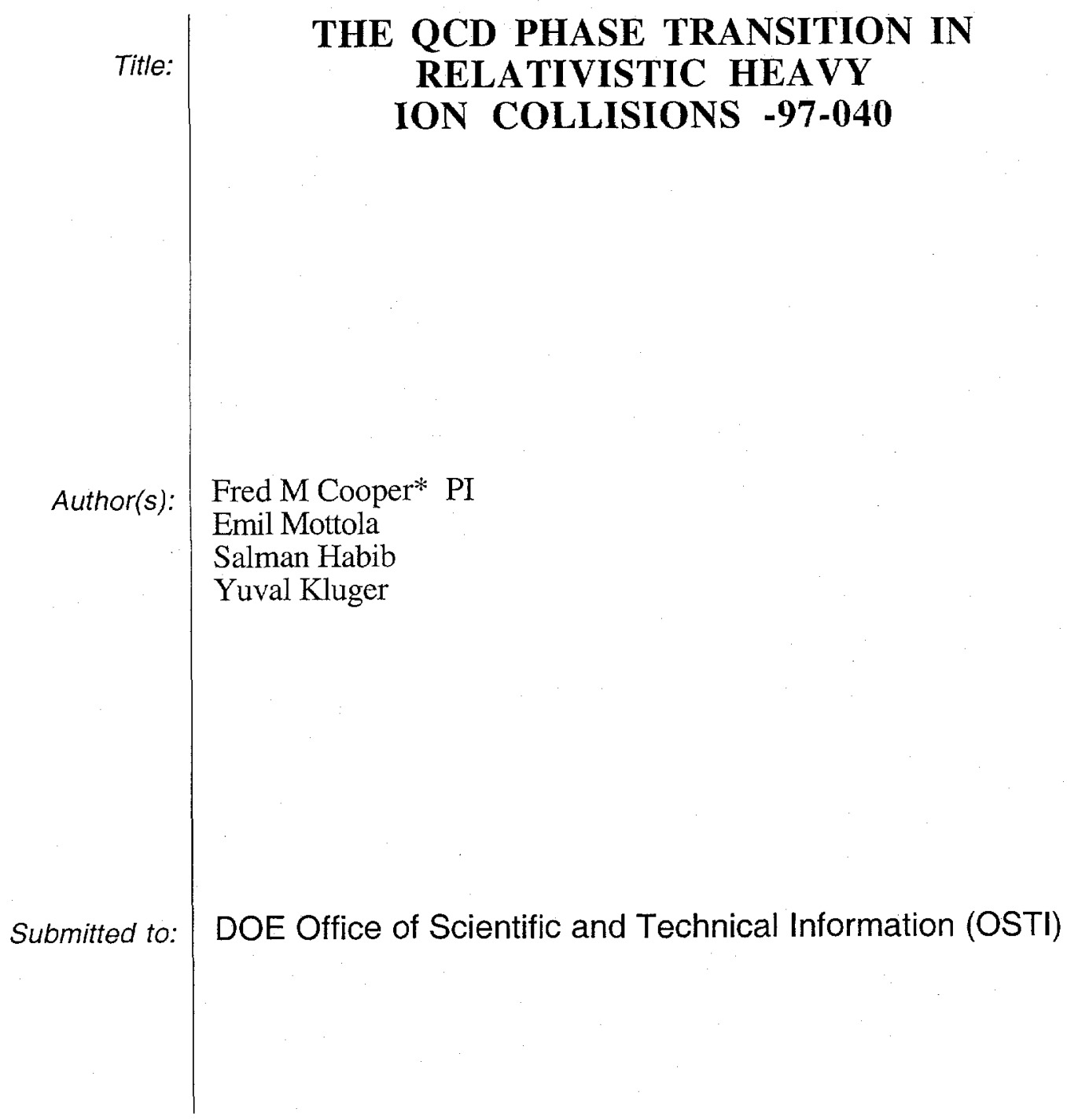

Los Alamos National Laboratory, an affirmative action/equal opportunity employer, is operated by the University of California for the U.S. Department of Energy under contract W-7405-ENG-36. By acceptance of this article, the publisher recognizes that the U.S. Government retains a nonexclusive, royaltyfree license to publish or reproduce the published form of this contribution, or to allow others to do so, for U.S. Government purposes. Los Alamos National Laboratory requests that the publisher identify this article as work performed under the auspices of the U.S. Department of Energy. Los Alamos National Laboratory strongly supports academic freedom and a researcher's right to publish; as an institution, however, the Laboratory does not endorse the viewpoint of a publication or guarantee its technical correctness. 


\section{DISCLAIMER}

This report was prepared as an account of work sponsored by an agency of the United States Government. Neither the United States Government nor any agency thereof, nor any of their employees, make any warranty, express or implied, or assumes any legal liability or responsibility for the accuracy, completeness, or usefulness of any information, apparatus, product, or process disclosed, or represents that its use would not infringe privately owned rights. Reference herein to any specific commercial product, process, or service by trade name, trademark, manufacturer, or otherwise does not necessarily constitute or imply its endorsement, recommendation, or favoring by the United States Government or any agency thereof. The views and opinions of authors expressed herein do not necessarily state or reflect those of the United States Government or any agency thereof. 


\section{DISCLAIMER}

Portions of this document may be illegible in electronic image products. Images are produced from the best available original document. 


\title{
The QCD Phase Transition in Relativistic Heavy Ion Collisions
}

\author{
Fred M. Cooper * \\ Emil Mottola \\ Salman Habib \\ Yuval Kluger
}

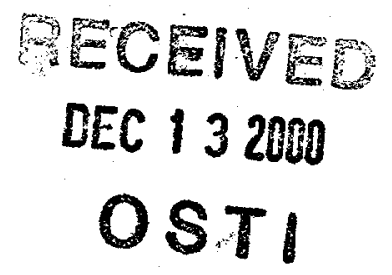

\begin{abstract}
This is the final report of a three-year, Laboratory-Directed Research and Development (LDRD) project at the Los Alamos National Laboratory (LANL). The objective of the research was to develop practical numerical methods using a first principles field theory approach to describe and study nonequilibrium phase transitions of nuclear and quark matter in ultrarelativistic heavy ion collisions at the Brookhaven Relativistic Hadron Ion Collider (RHIC). Our approach was based on a systematic $1 / \mathrm{N}$ expansion, and a density matrix formalism was developed to enable us to generate statistical ensembles of hypothetical experimental events. We performed numerical simulations of the chiral phase transition and determined experimental signatures such as distortion of pion and dilepton spectra as a result of the production of disoriented chiral condensates. We obtained an effective transport as well as hydrodynamic description of our mean field theory results. We found that a naive use of the $1 / \mathrm{N}$ expansion led to secular behavior and thus resummation methods were needed before being compared with experiment.
\end{abstract}

\section{Background and Research Objectives}

The objective of the research was to develop practical numerical methods using a first principles field theory approach to describe and study nonequilibrium phase transitions of nuclear and quark matter in ultrarelativistic heavy ion collisions at RHIC. At the beginning of our research, only mean-field methods were available to study this type of problem. To go beyond mean field theory we developed a $1 / \mathrm{N}$ expansion method using Schwinger's Closed Time Path formalism. We hoped this would allow us to determine important rethermalization times and give us more solid predictions for expermentalists at RHIC on how nonequilibrium effects distort pion and dilepton spectra from those expected in the local thermal equilibrium scenario then being assumed. On the computational side we hoped to be able to develop algorithms for solving inhomogeneous initial value problems so that various topological configurations could be studied and their effects understood.

*Principal Investigator, e-mail: fcooper@lanl.gov 


\section{Importance to LANL's Science and Technology Base and National R\&D Needs}

The research in this project uses methods that are related to those being developed to study nonequilibrium phenomena in general in the core competency area of nonequilibrium science. The RHIC accelerator at Brookhaven is the major DOE Nuclear Physics experimental physics facility for understanding the production of the quark-gluon plasma and the physics related to the evolution of the early universe. Our research was to understand the nonequilibrium aspects of the quark-gluon plasma expected to be formed during the ultrarelativistic heavy ion collisions at RHIC. In particular the laboratory has played a leading roll in the devleopment of the PHENIX detector that is one of the main detectors at the RHIC facility.

\section{Scientific Approach and Accomplishments}

Exact simulations of non-equilibrium phenomena in quantum field theory are not possible because the exact theory is equivalent to an infinite number of coupled nonlinear partial differential equations for the correlation functions describing the quantum fluctuations. Thus it is necessary to develop approximation methods where at lowest order the main features are captured. Mean field theory is a reasonable first approximation in that it describes the equilibrium phase structure of many theories quite well. The main problem has been to develop a systematic expansion around mean field theory which is well behaved and captures in low orders the major physics ingredients left out of the mean field calculation. During the time period of this proposal, we investigated an expansion around mean field theory based on a small parameter $1 / \mathrm{N}$ where $\mathrm{N}$ was the number of species of particles. In the context of mean-field theory we studied several effective field theories having the same chiral phase transition expected for the quark-gluon plasma. We found evidence that the non-equilibrium nature of the phase transition could effect the distribution of various particles produced following a heavy ion collision such as the spectrum of pions and dileptons. We were also able to give algorithms for studying inhomogeneous evolution of the plasma which would allow one to study various coherent phenomena such as textures, disoriented chiral condensates or vortices in plasmas. These algorithms are just being implemented in model field theories as this review is being written.

To understand the domain of validity of the expansion in $1 / \mathrm{N}$ we studied an exactly solvable quantum mechanics problem with $\mathrm{N}$ coupled anharmonic oscillators. 
In quantum mechanics we found a phase transition in the parameter $1 / \mathrm{N}$, making it unsuitable for simulations at modest $\mathrm{N}<20$. For $\mathrm{N}<20$, the expansion worked well at short times but then failed for times (measured in the oscillation frequency of a particle of mass $\mathrm{m}$ ) of order $\mathrm{N}$. This result then led us to explore methods of improving on the $1 / \mathrm{N}$ approximation. These improvements have been accomplished by us after the completion of this project. During the time-period of this project, other investigators showed that the phase structure of QCD had some new features at finite chemical potential-namely the existence of a superconducting phase where pairs of quarks form the order parameter.

We then switched our attention to producing a simple model with this property in order to study the dynamics of out of equilibrium phase transitions at finite chemical potential. Subsequent to the ending of this project we have been able to perform simulations of these phase transitions,

The achievements of the project were to perform the first out of equilibrium field theory evolution of the disoriented chiral condensates formed following a chiral phase transition. We also determined the distortion of the pion and dilepton spectra in a mean field approximation and published these spectra so that the experimentalists could look for these signatures. We also were able to give a consistent formalism for obtaining the evolution of inhomogeneous condensates in various quantum field theory settings. We were able to give the first derivation of how to obtain a quantum transport equation directly from a first principles field theory approach. We also invented a 4-Fermi model which had the same phase structure QCD at finite density and temperature which we subsequently have used to study phase transitions at non-zero baryon density. 


\section{Publications}

1. F. Cooper, S. Habib,Y. Kluger and E. Mottola, "Nonequilibrium Dynamics of Symmetry Breaking in $\lambda \varphi^{4}$ Field Theory" Phys.Rev. D55 6471-6503 (1997)

2. F. Cooper, Y. Kluger and E. Mottola "Anomalous Transverse Distribution of Pions as a signal for the production of DCC's" Phys. Rev. C54 3298 (1996)

3. F. Cooper, M. Kennedy and J. Dawson "Time evolution of the chiral phase transition during a spherical expansion" Phys.Rev. D54 2213 (1996).

4. F. Cooper "From Landau's Hydrodynamical Model to Field Theory Models of Multiparticle Production" in The State of Physics at the End of the 20th Century ed., F. Cooper et.al World Scientific (1999)

5. B. Mihaila, J. F. Dawson, F. Cooper, "Order $1 / \mathrm{N}$ corrections to the time dependent hartree approximation for a system of (N+1) oscillators", Phys.Rev. D56 5400$5412,(1997)$

6. B. Mihaila, J. F. Dawson, F. Cooper, M. Brewster, S. Habib, "The quantum roll in D-dimensions and the large D expansion". Aug 1998. 13pp. hep ph/9804288

7. D. Boyanovsky, F. Cooper, H.J. de Vega, P. Sodano "Evolution of inhogeneous condensates: selfconsistent variational approach". Phys. Rev.D58, 025007,(1998)

8. F. Cooper, "Unusual Dileptons at RHIC" in "Non-equilibuium many body Dynamics". Proceedings of the RIKEN BNL Research Center Workshop, Volume 4. BNL-64912. (1997).

9. V. Koch, J. Randrup, X.N. Wang, Y. Kluger, "Dileptons from disoriented chiral condensates". Nucl.Phys. A638, 447-450,(1998)

10. V. Koch, J. Randrup, Xin-Nian Wang, Y. Kluger, "Dileptons from disoriented chiral condensates". Proceedings of APCTP Workshop on Astro-Hadron Physics:

Properties of Hadrons in Matter, Seoul,Korea, 25-31 Oct 1997. e-Print Archive: nuclth/9712061

11. Y. Kluger, V. Koch, J. Randrup, Xin-Nian, "Dileptons from disoriented chiral condensates", Phys.Rev.C57, 280-290,(1998)

12. Y. Kluger, E. Mottola, J. M. Eisenberg, "The quantum Vlasov equation and its Markov limit", Phys. Rev.D58, 125015, (1998)

Abstract and Postscript from Los Alamos (or from France or Italy or U.K.)

13. G.F. Bonini, S. Habib, E. Mottola, C. Rebbi, R. Singleton, P.G. "Tinyakov, Periodic instantons in SU(2) Yang-Mills-Higgs theory".

BUHEP-99-10, May 1999. 12pp. e-Print Archive: hep-ph/9905243

14. F. Cooper, "Inclusive dilepton production at RHIC: A field theory based on a nonequilibrium chiral phase transition". BC-HEP-98-2, Nov 1998. 14pp. Phys. Rept.315, 59-81,(1999) e-Print Archive: hep-ph/9811246 
15. A. Chodos, H. Minakata, F. Cooper, "Cooper pairing at large $\mathrm{N}$ in a twodimensional model”. YCTP-P24-98, Phys.Lett.B449, 260-266,(1999) e-Print Archive: hep-ph/9812305

16. A. Chodos, F. Cooper, H. Minakata, "Competing condensates in twodimensions". YCTP-P7-99B, May 1999. Phys.Rev.Lett. 83 (1999) 1521-1524. e-Print Archive: hep-ph/9905521

17. A. Chodos, F. Cooper, W. Mao, H. Minakata, A. Singh "A Two-dimensional Model with Chiral Condensates and Cooper Pairs having QCD-like Phase Structure" Phys.Rev. D61 (2000) 045011, hep-ph/9909296

18. A. Chodos, H. Minakata, F. Cooper, "Inclusive Dilepton Production at RHIC". Phys. Rept. 315 (1999) 59-81

19. F. Cooper, "Initial Value Problems in Quantum Field Theory". hep-ph/9808234

20. B. Mihaila, T. Athan, F. Cooper, J.F. Dawson, S. Habib, "Exact and approximate dynamics of the quantum mechanical $\mathrm{O}(\mathrm{N})$ model". to be published. HEP-PH/0003105

\section{References}

1. B. Mihaila, F. Cooper, J.F. Dawson, "Resumming the large-N approximation for time evolving quantum systems". HEP-PH/0006254

2. A. Chodos, F. Cooper, W. Mao, A. Anupam, "Dynamics of the chiral phase transition at finite chemical potential in the Gross-Neveu Model". To be submitted to Phys. Rev. D LAUR-00-3104. 